erfolgreich. «Schmerz» scheint sich um jenes alte Dilemma nicht zu kümmern. Mein rasender Zahn treibt mich zum Zahnarzt.» [2]

Der Körper und seine Steuerung im Gehirn, als virtuelle Einheit betrachtet, ist einem einfach denkenden Haustier sehr ähnlich. Mit Tieren kann man bekanntlich nicht alles machen, mit Menschen aber schon. Im Gegensatz etwa zu Hunden kennen die Menschen ihre Grenzen kaum und lassen sich von tatsächlichen oder eingebildeten Autoritäten gewohnheitsmässig dazu verleiten, diese zu missachten. Diese Durchlässigkeit für Normen anderer Leute ist bei Kopfwehleidenden besonders ausgeprägt, sie sind dem "was würde man denn sagen, wenn ich das täte» oft hilflos ausgeliefert, und sie brauchen oft besondere Schutzmassnahmen dagegen, die die Notbremse Kopfweh nach und nach ersetzen. Am besten eignen sich dafür regelmässige Unterhaltsarbeiten am Körper, wie etwa tägliche Entspannungsübungen frühmorgens und tägliches längeres Gehen im Freien.

\section{Die Rolle des Alltäglichen und der Zeit in der Kopfwehpraxis}

Zur genügenden Erfassung des Kopfwehleidens gehört also die Erfassung des Alltagslebens der Patienten, die Beschreibung des Zeitmusters der Kopfschmerzen in bezug auf ihren Alltag, und der Vergleich der Entwicklung dieser Verhaltensmuster in der Zeit bis zur ersten Konsultation und während der Behandlungszeit. Wer Kopfweh ernsthaft behandeln will, kann nicht nur auf Medikamente vertrauen. Sie oder er wird sich die nötige Zeit schon nehmen oder gar stehlen müssen.

\section{Literatur}

1 Willis T. De Anima Brutorum, quae hominis vitalis ac sensitiva est, exercitationes duae. London: J. Allestry; 1672. Pars secunda, clinica, Beginn 1. Kapitel. Übersetzung: H. Isler.

2 Sherrington C. Man on his Nature. The Gifford Lectures. Edinburgh 1937-38. Cambridge: University Press; 1940. P. 226. Übersetzung: H. Isler.

\title{
Kopfschmerz als Alarmsignal
}

\author{
G. Jenzer
}

\begin{abstract}
"Alarm!?"
Alarm ist ein Zeichen, das vor unmittelbarer Gefahr warnt. Bei etwa bis 2\% aller medizinischen Notfälle ist das Symptom Kopfschmerz ausschlaggebend. 95\% dieser Patienten dürften dabei eine verhältnismässig harmlose Art von Kopfschmerz aufweisen; die verbleibenden $5 \%$ fallen jedoch unter die hier $\mathrm{zu}$ erörternde Thematik [1]. Kopfschmerz - einerseits neu oder schon länger vorhanden, andererseits episodisch, anfallsartig oder kontinuierlich - ist ein häufiges Symptom. Sowohl bei der ersten Begegnung mit dem Problem als auch bei Verlaufskontrollen sind wir also immer wieder wegen der Frage nach der potentiellen Gefährlichkeit möglicher Ursachen in die Verantwortung genommen. Hier zu klären, ist fast stets
\end{abstract}

Korrespondenz:

Dr. Gerhard Jenzer

Neurologie FMH

Jurastrasse 41

CH-4900 Langenthal

E-mail gjenzer@dial.eunet.ch auch ein dringliches, wenn oft unausgesprochenes Anliegen unserer Patienten. Bis man sich wegen Kopfschmerz untersuchen lässt, braucht es meist viel. Der entscheidende Beweggrund ist schliesslich in der Regel, ganz abgesehen vom Schmerz, Besorgnis oder gar eigentliche Angst. Es geht also darum, wann Kopfschmerz als solitäres Leitsymptom alarmierend ist. Um uns dieser alltäglichen Anforderung so sachgemäss wie nur möglich anzunehmen, wünschten wir uns zuverlässige Anhaltspunkte. Zum vornherein ist festzustellen, dass die Kopfschmerz-Diagnostik eine heikle, absolut exklusiv ärztliche Angelegenheit und - allen jenen banalisierenden Meinungsmachern zum Trotz - nicht Sache dilettantischer Anbieter auf dem Graumarkt des Gesundheitswesens sein kann.

\section{Diagnostisches Dilemma}

Bei etwa 90\% aller Kopfwehprobleme handelt es sich um sogenannte "primäre» Kopfschmerzen (v.a. Spannungskopfschmerz, Migräne, Cluster-Kopfschmerz, sowie konsekutiv chronische Kopfschmerzarten) [2], bei $10 \%$ also um "sekundäre" (symptomatische) und innerhalb denselben nur ausnahmsweise um unmittelbar gefährliche Kopfschmerzen. Stets jedoch, und ganz besonders bei der Erstuntersuchung oder bei unbefriedigenden Verläufen, muss somit eine Alarmbereitschaft, jedenfalls zumindest eine hohe Aufmerksamkeit vorhanden sein -, und eigentliche Alarmzeichen dürfen wir keinesfalls ignorieren. Wir übernehmen in jedem Einzelfall eine belastende Verantwortung und solange wir noch nichts vorfinden als das 
Symptom Kopfschmerz, plagt uns das diagnostische Dilemma.

Das Vorkommen sekundärer Kopfschmerzen kann hinsichtlich Ursachen [3] anteilmässig aufgegliedert werden. Dabei überwiegen bei weitem Kopfschmerzen im Rahmen von Infekten - etwa Grippe, Sinusitis - und sind dabei, abgesehen von eigentlichen Entzündungen an Gehirn und Meningen, meistens auch nicht gefährlich. Am wahrscheinlichsten handelt es sich sonst um Kopfschmerzen nach Kopftrauma oder bei Hirndurchblutungsstörungen verschiedener Art, wie insbesondere der akuten Subarachnoidalblutung. Monosymptomatisch handelt es sich verhältnismässig sehr selten (0,1\% der sekundären Kopfschmerzen), wenn auch meistens im Vordergrund der Befürchtungen, um Kopfschmerz bei Hirntumor. In mehr als 40\% der Fälle fehlte dieses Symptom präoperativ. Waren Schmerzen vorhanden, dann typischerweise progressiv, intermittierend ohne tageszeitliche Bindung, stundenlang anhaltend, jedoch nicht andauernd [4]. Vorhandensein von Kopfschmerz lässt somit aus diagnostischer Sicht keineswegs zuerst an einen endokraniellen Tumor denken.

Die meisten dieser Affektionen kommen natürlich nicht notwendigerweise als Notfälle zu uns, befinden sich aber als Arbeitshypothese zunächst stets im breiten differentialdiagnostischen Spektrum sekundärer Kopfschmerzen (Abb. 1: unvollständige Aufzählung, Gesichtsschmerz unberücksichtigt).

\section{Abbildung 1}

Sekundäre Kopfschmerzen

Folgen von Schädelhirntrauma

entzündliche ZNS-Erkrankungen (Meningitis, Enzephalitis)

endokranielle Druckerhöhung (Hirntumor, Pseudotumor cerebri, Liquorzirkulationsstörungen), Hypoliquorrhoe

- Hirnzirkulationsstörungen (Insulte, Subarachnoidalblutungen, Gefässdissektionen, Hirnvenen-/ Hirnsinusthrombosen)

Riesenzellarteriitis

maligne arterielle Hypertonie/Phäochromozytom

metabolische Störungen, Intoxikationen

Glaukom

\section{Schmerzentstehung "primär» - "sekundär»}

Als neuroanatomische Strukturen im peripheren Bereich sind hauptsächlich der N. trigeminus beteiligt sowie die hohen Zervikalwurzeln, welche zentral ebenfalls mit dem Trigeminuskerngebiet in Verbindung stehen. Hat die nozizeptive Information den Hirnstamm erreicht, werden weitere Anteile des ZNS (zentrales Höhlengrau und Thalamus) über Projektionen einbezogen, wonach höhere Zentren, unter Voraussetzung des Bewusstseins, Schmerzen zum Erleben bringen. Schmerzempfindliche Strukturen sind besonders die Meningealarterien, grössere Kopfarterien und venöse Sinus. Der modernen Migräneforschung, also weitgehend derjenigen an den Triptanen, verdanken wir einen Grossteil unserer heutigen, allgemeinen Kenntnisse über die Pathophysiologie der Kopfschmerzen. Die sogenannte «neurovaskuläre Theorie» beinhaltet die Vorstellung, dass Migräne im Gehirn beginnt und von dort über die Trigeminusinnervation die Kopfgefässe erreicht. Neueste Erkenntnisse sprechen dafür, dass auch der äusserst heftige, seitenkonstante Schmerz einer Clusterattacke "primär» in Gang gesetzt wird, wenn auch etwas anders als die Migräne, nämlich über eine Art Schmerzgenerator im Hypothalamus [5]. Die uns heute besser bekannt gewordenen primären Kopfschmerzen überlappen wahrscheinlich in einer sowohl anatomischen wie auch physiologischen Wechselbeziehung mit den sekundären Kopfschmerzen. Dies sehen wir beispielsweise daran, dass ein Hirninfarkt oder ein Meningeom durchaus typische Migräneanfälle triggern kann. Dasselbe gilt aber auch für Schmerzen etwa am Kiefer oder im Nacken, oder selbst weit vom Kopf entfernt. Primäre und sekundäre Kopfschmerzen laufen offenbar in gemeinsamen Systemen ab und weisen überdies zumindest teilweise identische "Mechanismen» auf. Daraus lässt sich folgern, dass die alleinige Betrachtung des Kopfschmerzes nur sehr bedingt über dessen eigentliche Herkunft, Entstehung, und somit Stellenwert Aufschluss geben kann. Insofern ist die Interpretation ausschliesslich der Schmerzsymptomatik für die Unterscheidung zwischen "primär» (prinzipiell harmlos) und "sekundär» (potentiell gefährlich) oftmals unzureichend. Zur Klärung dieser Frage stellen weitere anamnestische Gesichtspunkte sowie die eingehende physische Untersuchung absolut unverzichtbare Zusatzinformationen dar.

\section{Alarmierende Kriterien}

Trotz dieser Einschränkung kommt den Schmerzcharakteristika ganz wesentliche Aussagekraft zu. Alarmierende Attribute von Kopfschmerz sind: neu, unbekannt, ungewohnt, andauernd, zunehmend, schlagartig, explosiv, hohe Intensität, konstante Lokalisation, Auslösung durch Anstrengung und sexuelle Aktivität, nächtliches Auftreten. Die Intensität der Schmerzen - sozusagen also die Lautstärke der Alarmglocke - verhält sich vor allem im unteren Bereich keineswegs immer direkt proportional zur Grösse der anstehenden Gefahr. So können etwa ein chronisches Subduralhämatom oder ein Hirntumor u.U. kaum Kopfschmerz verursachen, oder dieses Symptom wird wegen mentaler Dysfunktion nicht mehr zureichend mitgeteilt. Allerdings ist jedoch ganz heftigster Schmerz - abgesehen von so typischen Ausnahmen wie dem Cluster-Kopfschmerz oder der Trigeminusneuralgie - meistens ein sehr starkes Indiz für eine bedrohliche Situation.

Nun einmal abgesehen vom Schmerz selbst sind eindeutige Alarmzeichen irgendwelche Merkmale eines zerebralen Ausfalls oder einer Fehlfunktion und in jedem Fall Meningismus. Die Alarmierung ist aber auch gegeben bei Erkrankung und speziell Fieber sowie nach Unfällen, selbst wenn die initiale Kopfbeteiligung nicht offensichtlich war (z. B. bei Antiko- 
agulierten mit der Möglichkeit einer endokraniellen Blutung). Stets sind also sämtliche verfügbaren klinischen Elemente einzubringen und sorgfältig abwägend im medizinischen Gesamtzusammenhang zu verwerten.

Unter den uns zur Verfügung stehenden Mitteln ist die Anamnese entscheidend, wenn auch oft mit erheblichem Zeitbedarf verbunden. Dafür macht sie schätzungsweise 90\% des Abklärungsbeitrages aus. Als zweitwichtigster Teil folgt die allgemeine Untersuchung mit Neurostatus und - obligat - die Prüfung des Meningismus. In ausgewählten Fällen braucht es Zusatzuntersuchungen wie Labor und insbesondere Neuroradiologie. Als Screeningmethode ist das Schädel-CT rasch verfügbar, in der Routinediagnostik allerdings ohne komplette Voruntersuchungen eher unergiebig. Bei mehr als 3000 Abklärungsfällen einer amerikanischen Kopfwehklinik erbrachte das Schädel-CT recht geringe Prozentzahlen von positiven Befunden, nämlich zwischen 1,2 und 0,2\% für die Hauptgruppen (in absteigender Häufigkeit): Hirninfarkte, Tumore, Aneurysma und Angiome, Hydrozephalus und Subduralhämatom [6]. «CT-negativer Kopfschmerz" enthebt nicht von einer differentialdiagnostischen Einordnung, ohne welche der Behandlungserfolg von Anfang an kompromittiert bleibt.

\section{Entscheidungsraster}

Eventuell liegt eine von Anfang an klare Notfallsituation vor. Zuweilen darf ein Entscheid auch vorsichtig, unter strikter Beobachtung vertagt werden. Ist allerdings ein Verdacht bereits vernünftig begründet, wollen wir nicht weniger Abklärungsaufwand, sondern mehr Information! In jedem Zweifelsfall ist die Weiterweisung oder Hospitalisation allemal einem spekulativen Abwarten vorzuziehen - und dies nicht nur zum Wohl des Patienten, sondern auch zum Schutz seiner ärztlichen Helfer.

Für den Entschluss zur weiteren Abklärung unter dem Gesichtspunkt einer mehr oder weniger unmittelbaren Gefährlichkeit, sei es Zuweisung an Spezialisten oder zunächst zur Neuroradiologie, brauchen wir einen möglichst eindeutigen Entscheidungsraster. Ist nur ein einziger der untenstehend aufgeführten Punkte zutreffend, so empfiehlt sich das Weitergehen (Abb. 2).

\begin{tabular}{l}
\hline Abbildung 2 \\
\hline Wann abklären bei unklarem Kopfschmerz? \\
- schlimmster Kopfschmerz ("first and/or worst») \\
- epileptische Anfälle, neurologische Funktionsstörungen \\
- Bewusstseinsstörung oder sonstige mentale Dysfunktion \\
- blosser Verdacht auf Meningismus \\
- geringste Abweichung im Neurostatus \\
- allgemeine Krankheitszeichen/Fieber \\
- Schmerzzunahme \\
- Verschlechterung der Gesamtsituation
\end{tabular}

Cum grano salis kann mit weiteren Schritten beobachtend zugewartet werden, wenn folgende Punkte ohne Ausnahme erfüllt sind: Es handelt sich um einen lang bekannten und im Wesen unveränderten Kopfschmerz. In der Vorgeschichte fehlen Hinweise auf epileptische Phänomene oder sonstige vorübergehende Funktionsstörungen zerebralen Ursprungs. Bewusstseinslage, Neurostatus, Prüfung auf Meningismus und Allgemeinuntersuchung sind normal, einschliesslich Afebrilität. Und schliesslich darf im weiteren Verlauf keinerlei Verschlechterung auftreten [7].

\section{«First and/or worst ever!»}

Als besonderes verdächtig für gefährlichen Kopfschmerz gilt die unmittelbare Provokation eines sehr rasch auftretenden Kopfschmerzes unter körperlicher Anstrengung oder bei sexueller Aktivität. Gerade oftmals in einem solchen Zusammenhang, durchaus jedoch auch ohne erkennbaren Grund, kann erstmaliger heftigster Kopfschmerz auftreten, welcher als Warnkopfschmerz ("sentinel headache») einmalig oder wiederholt einer akuten Subarachnoidalblutung um Minuten, Stunden oder bis mehrere Wochen vorangeht. Es ist zu hoffen, dass ein solches Ereignis sowohl den Betroffenen zum Gang in die Arztpraxis bewegt, als auch dort erkannt und zum Anlass für eine Weiterabklärung genommen wird. Dieser Schmerz entsteht bei Hirnarterienaneurysma durch begrenzte Einblutung ("Warnblutung») in die Gefässwand oder in die unmittelbare Umgebung. Ungünstigenfalls kommt es später zur eigentlichen akuten Subarachnoidalblutung mit einem meist urplötzlichen, vernichtenden und schlagartigen Kopfschmerz, ("thunderclap headache»). Ausschlaggebend ist die Schilderung, dass es sich um einen bisher nie gekannten, unvorstellbar intensiven Schmerz handelt ("the first and/or worst ever!»). Fatalerweise, wenn auch selten, kann der Kopfschmerz aber auch schwach ausfallen oder, zumindest scheinbar, überhaupt fehlen. Zum Bild gehören - ähnlich dem Migräneanfall - Nausea, Photophobie und Erbrechen, zusätzlich aber auch ein Meningismus. Eine Bewusstseinsstörung, allenfalls bis zum Koma, findet sich bei 25\% dieser Fälle, ein pathologischer Neurostatus (Halbseitensymptome, Hirnnervenausfälle) ebenfalls bei 25\%. Bei bis etwa 50\% aller Fälle ist ein Warnkopfschmerz vorangegangen [8]. Die Mortalität der ersten Blutung liegt hoch (um 40\%) und Fehldiagnosen sind häufig. Am besten erfolgt die Einweisung gerade in die Spezialklinik. Innert der ersten 24 bis 48 Stunden ist der Nachweis der Aneurysmablutung mit dem Nativ-CT in 95\% der Fälle möglich. Bei negativem Resultat ist Insistieren mit Liquorpunktion zum Nachweis von frischem Blut und/oder Xanthochromie zwingend. Ein erstmaliger und/oder zugleich schwerster Kopfschmerz ist also mit Priorität und bis zum Beweis des Gegenteils verdächtig auf ein Hirnarterienaneurysma bzw. eine akute Subarachnoidalblutung. Fehlt dieser Nachweis, so kann, didaktisch problematisch genug, ein benigner/idiopathischer 
"thunderclap headache» [9] in Betracht gezogen werden. Manche andere Affektionen fallen in die Differentialdiagnose, wie u.a. zerebrale Embolie, Hirntumor, endokranielle Hämatome, arteriovenöse Missbildungen, Meningitis und Dissektionen grosser Halsgefässe. Der Stellenwert und die Symptomatik der Karotis- und Vertebralisdissektionen ist erst in den letzten Jahren ausreichend erkannt und beschrieben worden. Auch hier kann eine Frühdiagnose aus therapeutischen Gründen (Vollantikoagulation) entscheidend sein. Wenn auch die bei etwa $3 / 4$ der Betroffenen auftretenden Kopfschmerzen zufolge Dissektion grosser Halsarterien nicht derart kennzeichnend sind wie bei der akuten Subarachnoidalblutung zufolge Aneurysmaruptur, so weisen sie doch charakteristische Merkmale auf. Sie sind meistens heftig und homolateral lokalisiert: bei der Karotisdissektion periorbital, frontal, temporal und anterolateral am Hals; bei der Vertebralisdissektion okzipitonuchal sowie posterolateral am Hals [10]. Eine Kopfschmerzzeichnung kann dies deutlich machen. Zur Verdachtsdiagnose gehören aber auch typische Begleitsymptome und neurologische Ausfälle.

\section{Echter und falscher Alarm}

Selbst unter minutiöser Beachtung aller Kriterien verbleibt eine Rest an Ungewissheit. Migräneartiger Kopfschmerz als Ausdruck einer Erkrankung und primäre Migräneanfälle sind unter kurzfristiger Beobachtung diagnostisch nicht immer einwandfrei trennbar. Selbst langdauernder, unveränderter Kopfschmerz bietet keine Gewähr für Unbedenklichkeit. Das Alarmzeichen Kopfschmerz kann aber auch gänzlich ausfallen oder der Beobachtung entgehen, etwa bei Vorliegen einer dominierenden sonstigen Affektion und ganz besonders bei manchmal nicht leicht feststellbarer mentaler Veränderung; schliesslich kann es aber überhaupt fehlen (z.B. bei einem Meningeom).
Kopfschmerz kann echten Alarm bedeuten und eine gefährliche oder potentiell gefährliche Affektion ankündigen, ist also auch als Einzelsymptom zunächst niemals unbedenklich. Abgesehen vom "sentinel headache" und dem "thunderclap headache», sowie allenfalls vom Kopfschmerz bei Dissektion der grossen Halsgefässe, kennen wir kaum einen kennzeichnenden Alarmkopfschmerz. Wir können somit wegen einer bestimmten Art von Kopfschmerz meistens nicht vorweg entscheiden, ob es sich um etwas Gefährliches handelt. Insofern kann auch ein primärer Kopfschmerz zunächst als Alarmzeichen fehlgedeutet werden. Trotz einer bedrohlichen Situation finden wir manchmal normale Untersuchungsbefunde, so dass stets sämtliche verfügbaren Kenntnisse über die Begleitumstände sorgfältig in die Evaluation einzubeziehen sind. Nicht so selten gibt es wegen Kopfschmerz falschen Alarm, was eben in der Natur der Sache liegt.

\section{Literatur}

1 Swanson J, Winner P. Headache diagnosis and treatment in the emergency department. AAN, 51st Annual Meeting, Toronto, ON, Canada. Syllabi on CD-ROM, MARATHON MULTIMEDIA, North Field, MN, USA, 1999.

2 Jenzer G. Spannungskopfschmerzen und Migräne Management in der Praxis. Schweiz Med Wochenschr 1997; 127:1667-70.

3 Rasmussen BK, Olesen J. Symptomatic and nonsymptomatic headaches in a general population. Neurology 1992;42: 1225-31.

4 Pfund Z, Szapary L, Jaszberenyi F, Nagy F, Czopf J. Headache in intracranial tumors. Cephalalgia 1999;19:787-90.

5 Diener HC. Positron emission tomography studies in headache. Headache 1997;37:622-5.

6 Evans RW. Diagnostic testing for the evaluation of headaches. Neurol Clin 1996;14:1-26.

7 Edmeads J. Challenges in the diagnosis of acute headache. Headache 1990; 30(Suppl 2):533-40.

8 Schievink WI. Intracranial aneurysms. N Engl J Med 1997; 336:28-40.

9 Linn FHH, Rinkel GJE, Algra A, van Gijn J. Follow-up of idiopathic thunderclap headache in general practice. J Neurol 1999;246:946-8.

10 Sturzenegger M, Steinke W. Dissektionen der Hirnarterien. Ther Umschau 1996;53:544-51. 